\title{
LA PRESIDENCIA ACORRALADA DE ENRIQUE PENA NIETO
}

\section{THE ENCIRCLED PRESIDENCY OF ENRIQUE PEÑA NIETO}

\section{LA PRÉSIDENCE COINCÉE D’ENRIQUE PEÑA NIETO}

\author{
SOLEDAD LOAEZA \\ El Colegio de México \\ maloa@colmex.mx
}

Resumen: El presente artículo analiza la presidencia de Enrique Peña Nieto en el contexto más general de las transformaciones experimentadas por el presidencialismo mexicano como resultado de la apertura del sistema político y la creciente pluralidad observada, particularmente, desde la década de 1990. La hipótesis central es que el proceso de desmantelamiento del Estado autoritario y las reformas estructurales, despojaron la presidencia de la República de instrumentos para intervenir en la política y en la economía. La principal consecuencia de estos cambios fue el debilitamiento del poder ejecutivo, la creciente influencia de los intereses particulares y el impulso a la personalización de la presidencia.

Estas tendencias tuvieron una expresión particular durante los años de Peña Nieto en el poder, en los cuales se hizo evidente la falta de fortaleza del Ejecutivo. El trabajo examina en detalle el proceso y las dinámicas que llevaron a esta reconfiguración de la presidencia mexicana, y se concentra en destacar el efecto que durante el sexenio 2012-2018 esto generó en cuanto a la dificultad para contener a la violencia criminal y la corrupción, así como respecto de la importancia central adquirida por la personalización del puesto.

Palabras clave: presidencia de la República; México; Enrique Peña Nieto;

Partido Revolucionario Institucional; democratización.

Abstract: This article analyzes the presidency of Enrique Peña Nieto in the broader context of the transformations undergone by Mexican presidentialism as the result of the opening up of the political system and the growing plurality, particularly since the 1990s. The central hypothesis is that the process of dismantling the authoritarian state and structural reforms took away powers from the Office of the President to intervene in politics and the econ- 
omy. The principal consequence of these changes was the weakening of the Executive power, the growing influence of private interests and a shift towards a focus on the personality of the president.

These tendencies were expressed in a particular manner during Peña Nieto's term in office, revealing the Executive's lack of strength. This article examines in detail the process and the dynamics that led to this reconfiguration of the Mexican presidency, and highlights the resulting effects during the 2012-2018 administration, particularly with regard to the difficulty of containing criminal violence and corruption, as well as the personalization of the position of president.

Keywords: President's Office; Mexico; Enrique Peña Nieto;

Institutional Revolutionary Party; democratization.

\section{Traducción de Gonzalo Celorio Morayta}

Résumé: Cet article analyse le mandat d'Enrique Peña Nieto dans l'atmosphère des changements généraux qu'a subis le régime présidentiel mexicain à la suite de l'ouverture du système politique, laquelle a permis une pluralité croissante que l'on a pu constater surtout depuis les années 1990. L'hypothèse principale est que le processus de démantèlement de l'État autoritaire et les réformes structurelles ont dépouillé la présidence de la République des outils dont elle se servait jadis pour intervenir dans la politique et dans l'économie. L'effet principal de tels changements a été d'affaiblir le pouvoir exécutif, ce dont découlent une influence chaque fois plus grande des intérêts privés et l'élan pour personnaliser la présidence. Ces tendances se sont manifestées d'une façon marquée sous le gouvernement de Peña Nieto, le long duquel la perte de solidité de l'exécutif a été mise en évidence. L'article propose un examen détaillé des processus et des forces qui ont transformé ainsi la présidence mexicaine, au prix d'aggraver les difficultés à contenir la violence criminelle et la corruption entre 2012 et 2018, alors que la personnalisation de la fonction lui accordait une importance centrale.

Mots clefs: Présidence de la République; Mexique; Enrique Peña Nieto;

Partido Revolucionario Institucional; démocratisation.

\section{Traducción de Bernardo Mabire}

Fecha de recepción: febrero de 2020

Fecha de aceptación: marzo de 2020 
$\mathrm{D}$ urante décadas el presidencialismo mexicano fue ejemplo de un poder ejecutivo desbordado, cuyo peso se imponía al principio del equilibrio de pesos y contrapesos que debía gobernar el orden político. Este desequilibrio se alteró de manera significativa como resultado de las reformas liberales de los años noventa que transformaron de manera radical el perfil del Estado. El objetivo de los cambios que se introdujeron en las funciones estatales no era el poder del presidente, sin embargo, indirectamente lo reconfiguraron.

La presidencia de la República siguió siendo el eje del sistema político, pero la asimetría que antes caracterizaba las relaciones entre el Poder Ejecutivo, el Legislativo y el Judicial se corrigió parcialmente a expensas de la institución presidencial. Despojado de su aparente omnipotencia, el presidente enfrentó el desafío, en particular, del Congreso. En el periodo entre 1997 -cuando el Partido Revolucionario Institucional (PRI) perdió la mayoría en la Cámara de Diputados- y 2018, las relaciones entre el Poder Ejecutivo y el Legislativo se caracterizaron por una aguda tensión y una rivalidad que dificultaba la colaboración entre poderes y que, en más de un caso, hubiera podido paralizar al gobierno. En este contexto, Enrique Peña Nieto, candidato presidencial del PRI en 2012, fue elegido presidente.

Contrariamente a lo que muchos votantes del PRI esperaban, el regreso de ese partido al poder no fue una simple restauración; las condiciones institucionales y políticas del país habían cambiado de manera muy significativa durante los doce años que ese partido estuvo en la oposición.

En ese lapso el gobierno estuvo en manos del Partido Acción Nacional (PAN), que continuó la línea programática que habían puesto en práctica los presidentes Carlos Salinas y Ernesto Zedillo. Esto significa que llegó realmente a su fin la tradición del Estado jacobino, intervencionista y centralizador que había formado la Revolución. ${ }^{1}$ Una consecuencia de

${ }^{1}$ Soledad Loaeza, "La metamorfosis del estado. Del jacobinismo centralizador a la fragmentación democrática” (pp. 23-70), en Soledad Loae- 
esta reducción del Estado fue que el rasgo sobresaliente de la presidencia de Enrique Peña Nieto fuera la debilidad. En los años que van de 2012 a 2018, la fragmentación del poder en todos los niveles e instancias puso en riesgo la continuidad del sistema político; la situación era realmente muy grave desde el punto de vista de la seguridad interna, dada la dramática expansión de organizaciones criminales de grandes dimensiones, extremadamente violentas, poseedoras de inmensos recursos financieros y de armamento muy sofisticado. ${ }^{2}$ En más de tres décadas, el Estado mexicano ha tenido grandes dificultades para organizar y formar policías eficaces; recurrió al ejército, pero aun así, en general, el sistema de seguridad es muy pobre.

Desde inicios del siglo xxi, el Estado ha demostrado grandes limitaciones en el combate al narcotráfico. No sólo perdió la capacidad de articular y representar un interés colectivo porque las reformas liberales lo despojaron de los instrumentos que le permitían cumplir esa función, tampoco pudo mantener y garantizar el orden público de manera efectiva. En esa condición de debilidad, la presidencia de la República quedó a merced de diferentes grupos de interés, fundamentalmente empresariales que, a través suyo, capturaron el Estado y lo utilizaron en beneficio propio.

Este artículo desarrolla la hipótesis de que el proceso de desmantelamiento del Estado autoritario debilitó la presidencia de la República. Carente de instrumentos para intervenir en la política y en la economía, el Estado perdió autonomía. La disminución del poder estatal propició la creciente influencia de los intereses particulares y favoreció la expansión de la corrupción, porque los controles tradicionales se abandonaron y no se formularon nuevas reglas para

za y Jean-François Prud-homme (coords.), Los grandes problemas de México, México, El Colegio de México, 2010, tomo xiv.

${ }^{2}$ Jorge Chabat, "La respuesta del gobierno de Calderón al desafío del narcotráfico: entre lo malo y lo peor" (pp. 21-40), en Arturo Alvarado y Mónica Serrano (coords.), "Seguridad nacional y seguridad interior", Los grandes problemas de México, México, El Colegio de México, 2010, tomo xv. 
proteger a la institución presidencial de los efectos negativos del cambio. Asimismo, la debilidad del Estado favoreció la personalización exacerbada de la presidencia de la República, que quedó expuesta a las presiones de los intereses particulares.

Como se verá, ni siquiera la firma del Pacto por México, que reunió a las principales fuerzas políticas del país, en marzo de 2013, contribuyó a recuperar la fuerza del Estado o la autoridad de la presidencia de la República. Entre 2012 y 2018, en México ocurrió lo que Giovanni Sartori observó en los regímenes presidenciales: una oscilación constante entre el abuso del poder y la deficiencia del poder. ${ }^{3}$

Para desarrollar la hipótesis relativa a los orígenes de la debilidad de la presidencia mexicana, primero planteo el tema del presidencialismo en procesos de transición desde una perspectiva general; después se describen las reformas liberales que introdujeron los presidentes Miguel de la Madrid (1982-1988), Carlos Salinas (1988-1994) y Ernesto Zedillo (1994-2000). La tercera parte está dedicada a examinar el impacto de estos cambios sobre el PRI, que era un instrumento clave del ejercicio de la presidencia autoritaria; por último, describo la personalización de la presidencia de la República.

\section{Presidencialismo y Democratización}

El fortalecimiento del liderazgo político de la institución presidencial fue un efecto paradójico del proceso de democratización, y resultado del papel central que desempeñaron los presidentes como proponentes e impulsores del cambio. Iniciativas presidenciales y el apoyo renuente del PRI guiaron la trayectoria del reformismo político del siglo xx mexicano.

${ }^{3}$ Giovanni Sartori, Ingeniería constitucional comparada. Una investigación de estructuras, incentivos y resultados, México, Fondo de Cultura Económica, 1994, en especial el posfacio: "La transición de México ¿Hacia dónde? (Una agenda para la reforma)”, pp. 97-101. 
Especialistas, estudiosos y observadores no se ponen de acuerdo respecto a la fecha de inicio de la transición en México. Se ha atribuido a diferentes acontecimientos: el movimiento estudiantil de 1968, la reforma electoral de 1977, la escisión del PRI en 1987, la derrota del PRI en las elecciones federales de 1997, entre otros. El desacuerdo prevalece porque, a diferencia de lo que ocurrió con la mayoría de los regímenes autoritarios que en los años ochenta y noventa experimentaron una transición a la democracia, el régimen mexicano no se desplomó súbitamente y tampoco se vino abajo por la presión de movilizaciones en defensa de la democracia o por el triunfo de las oposiciones.

La democratización mexicana fue más bien el resultado de un largo proceso de desmantelamiento de instituciones y mecanismos autoritarios cuyo objetivo central era el control de la participación. La elite político-administrativa del periodo autoritario y el PRI, encabezados por los sucesivos presidentes, incluido José López Portillo, tuvieron un papel importante en esta transformación, y por momentos fueron más activos que las oposiciones.

El principal instrumento de democratización fue la legislación electoral que a partir de 1977 se reformó en repetidas ocasiones, parcial o íntegramente, con el fin de institucionalizar la participación política y para responder a las demandas de una ciudadanía que buscó sumarse a la ola antiautoritaria del último tercio del siglo xx.

La democratización no fue la causa inmediata del fin del presidencialismo autoritario; el desplazamiento de la presidencia de la República dentro de la estructura del poder político se inició desde la crisis que provocó la expropiación de la banca, que decretó José López Portillo el $1^{\circ}$ de septiembre de 1982. Esa medida, producto de la desesperación que le causaba al presidente una situación financiera crítica, sembró en la opinión pública una gran desconfianza hacia la arbitrariedad con la que ejercía el poder, que le permitía tomar una decisión de esa magnitud de manera unilateral. 
La protesta en contra de este ejercicio abusivo del poder se canalizó por la vía de movilizaciones contra el fraude electoral y la demanda de elecciones limpias y equitativas. En los años ochenta, estas protestas reclamaban el derecho a la participación política; fueron movilizaciones que buscaban recuperar el voto que a muchos les había sido expropiado con los fraudes del PRI.

De hecho, el fin del autoritarismo y el cambio político perseguían objetivos en apariencia limitados. No se trataba de instaurar un nuevo régimen; la democratización consistía simplemente en cumplir los ordenamientos constitucionales: el respeto a la división de poderes, el acotamiento del poder del presidente, y la inviolabilidad de las libertades fundamentales del individuo.

La trayectoria de varios países que hace más de una década lucharon por la democratización, y que ahora están gobernados por presidentes que actúan como los del pasado autoritario, apunta a que esta forma de gobierno tiene ciertas fallas que representan un riesgo para la consolidación de la experiencia democrática. Así Bolivia, Ecuador, Nicaragua, Perú y Venezuela evolucionaron hacia regímenes presidenciales muy semejantes a los que gobernaban estos países antes de la democratización. El problema no parece ser la rigidez de la que hablaba Juan Linz, sino que la legitimidad democrática con la que ahora llegan al poder se utiliza como coartada para acumular poder o mantenerse en éste indefinidamente.

La creencia de que el presidencialismo no es en sí mismo un régimen de opresión, sino que puede ofrecer incluso una vía de democratización, está anclada en una arraigada tradición constitucional de la región. Sin embargo, en algunos casos, el funcionamiento cotidiano de las instituciones del régimen presidencial democrático se topó con obstáculos significativos. En primer lugar, la persistencia de patrones de comportamiento y de actores provenientes del régimen autoritario; luego, la pobreza, el crecimiento económico mediocre o un arreglo institucional deficiente. La intervención de estos factores fue tan importante, que le restó precisión a la 
línea que separa cierto tipo de regímenes autoritarios del presidencial, de suerte que un político ambicioso puede cruzarla en cualquier momento, fincar un gobierno hiperpresidencialista y poner fin a la democratización. ${ }^{4} \mathrm{~A}$ inicios del siglo XXI, al abrigo del régimen presidencial, el autoritarismo se reinstaló en América Latina.

Así, y no obstante la reiteración del acto democrático por excelencia que es el voto, la consolidación del nuevo régimen puede ser interrumpida, si no es que pospuesta indefinidamente. Los ejemplos mencionados también apuntan a que la restauración antidemocrática ocurre en un momento de transición, cuando el arreglo posautoritario carece de mecanismos de defensa contra las inercias y las resistencias del régimen anterior. En estos vacíos institucionales, con frecuencia generados por el mismo proceso de transición, y que por lo mismo se piensan transitorios, la persona que ocupa la jefatura del Poder Ejecutivo dispone de un margen de autonomía amplio. En estas circunstancias, para compensar la debilidad institucional, puede recurrir a la personalización del poder, que es tanto una alternativa como una tentación.

Dada la asociación del presidencialismo con el sistema autoritario que se estableció en el México posrevolucionario, se hubiera esperado una revisión profunda del régimen político. Sin embargo, en México, como en los demás países de América Latina donde se produjo una crisis del autoritarismo, el régimen presidencial nunca fue verdaderamente cuestionado, salvo por algunos académicos. Además, el régimen presidencial se ha extendido a África, y varios países del antiguo bloque socialista optaron por esa forma de gobierno después de la caída del Muro de Berlín. ${ }^{5}$

${ }^{4}$ Adam Przeworski, luego de una amplia revisión histórica de la estabilidad de la democracia en el mundo, plantea que los regímenes presidenciales son particularmente vulnerables a los efectos de crisis económicas o políticas. Adam Przeworski, Crises of Democracy, Nueva York, Cambridge University Press, 2019, pp. 33-36.

${ }^{5}$ Raymond Huard, L'éléction présidentielle au suffrage universel, París, La Dispute, 2003. 


\section{LA DELGADA LÍNEA ENTRE PRESIDENCIALISMO Y AUTORITARISMO}

A mediados de los años ochenta, Juan Linz lanzó el debate acerca de las ventajas y desventajas del presidencialismo frente al parlamentarismo. ${ }^{6}$ Según el distinguido politólogo, el régimen presidencial tenía un fuerte potencial desestabilizador porque sujetaba al sistema político a rigideces, por ejemplo, los periodos de duración de mandato, que impedían ajustes que con frecuencia demandaban las cambiantes condiciones políticas. Por consiguiente, desde su perspectiva, el presidencialismo era en sí mismo un peligro para la democracia. La discusión perdió urgencia antes de que se hubiera llegado a un consenso.

\section{EL PRESIDENCIALISMO MEXICANO}

La explicación y el análisis del presidencialismo mexicano han estado dominados por un paradigma que establece dos dimensiones: la primera es que se trata de una forma personalizada de ejercicio del poder, que desborda a la institución presidencial. Tanto así que Enrique Krauze, el promotor más notable de esta visión, afirma que la biografía de los presidentes mexicanos es la biografía del poder. ${ }^{7}$ La segunda dimensión se refiere al alcance de ese poder que, según el paradigma, es ilimitado. ${ }^{8}$ Daniel Cosío Villegas planteó el paradigma en los siguientes términos: "Puesto que el presidente de México tiene un poder inmenso, es inevitable que lo ejerza personal y no institucionalmente, o sea que resulta fatal que la perso-

${ }^{6}$ Juan J. Linz y Arturo Valenzuela (eds.), The Failure of Presidential Democracy, Baltimore y Londres, The Johns Hopkins University Press, 1994, 2 vols.

${ }^{7}$ Enrique Krauze, La presidencia imperial. Ascenso y caída del sistema político mexicano, (1940-1996), México, Tusquets, 1997, p. 16.

${ }^{8}$ Soledad Loaeza, A la sombra de la superpotencia. Tres presidentes mexicanos en la Guerra Fría, (en prensa). 
na del presidente le dé a su gobierno un sello peculiar, hasta inconfundible". 9

Una de las premisas básicas de este artículo sostiene que, contrariamente a lo que afirma el paradigma del presidencialismo mexicano, la sola personalidad del individuo no basta para explicar su forma de gobernar ni las decisiones que toma.

Pese a todo, el contexto institucional determinaba recursos y restricciones, imponía reglas de comportamiento y límites que daban forma a la acción presidencial. La presidencia de la República estaba en el centro de la estructura del poder, centralizaba los recursos políticos de la sociedad y era el principal agente de modernización económica y política del país. La Constitución de 1917 le había atribuido facultades y recursos para llevar a cabo esas funciones. Los presidentes mexicanos tenían, como otros de distintos países de similar estructura, el poder de destruir, consolidar o crear instituciones. ${ }^{10}$ Tenían algunos instrumentos para contener los efectos de sus decisiones, pero eran insuficientes. Estos rasgos distintivos fueron poderosos recursos en la construcción de la hegemonía del PRI, entre 1946 y 1958, pero a partir de la década de los años ochenta pasaron a ser prueba del carácter antidemocrático del régimen.

Si la presidencia de la República no hubiera tenido una base institucional, si hubiera sido únicamente un instrumento para el ejercicio de un poder personal, la institución no habría sido factor de equilibrio del sistema político, como lo fue por lo menos hasta 2000, y tampoco habría sostenido la estabilidad política, como lo hizo durante la mayor parte del siglo xx. La presidencia de Enrique Peña Nieto ofrece un ejemplo ilustrativo de esta relación y de su impacto sobre procesos generales más amplios.

${ }^{9}$ Daniel Cosío Villegas, El estilo personal de gobernar, México, Cuadernos de Joaquín Mortiz, 1974, p. 8.

${ }^{10}$ Stephen Skowronek, The politics presidents make. Leadership from John Adams to Bill Clinton, Cambridge, The Belknap Press of Harvard University Press, 1993. 
El tipo de presidencialismo que se desarrolló en México a partir de 1945 había perdido los rasgos caudillescos que le había impuesto el origen revolucionario del sistema político, pero había conservado dos características derivadas del origen militar de la conquista del poder de la elite revolucionaria: una noción de la autoridad que la entendía como la subordinación al "jefe" que ejercía el poder de manera arbitraria; ${ }^{11}$ y una decidida intolerancia frente a la oposición.

El presidencialismo autoritario de los años de auge del PRI era una combinación de instituciones y de mecanismos propios de los regímenes sultanísticos y de los regímenes democráticos. Por una parte, su característica central era la discrecionalidad, que permitía al presidente actuar por encima de la ley y, por la otra, la organización constitucional del Estado se fundaba en la voluntad popular, la separación de poderes, el federalismo y el municipio libre. Estas dos dimensiones se materializaban en poderes formales y poderes informales que tendían a imponerse a los primeros. El peso de las facultades informales que asumía el presidente fortalecía el potencial de personalización del poder y, en no pocas ocasiones, los rasgos de carácter, las actitudes y las convicciones del señor presidente invalidaban las disposiciones constitucionales.

No obstante las apariencias, la presidencia no tenía el poder ilimitado que le atribuían los críticos. Era débil en comparación con otros regímenes presidencialistas porque la facultad de iniciativa legislativa no era exclusiva, tampoco podía emitir decretos de emergencia ni promulgar parcialmente una ley. ${ }^{12}$

${ }^{11}$ En un ensayo sobre el mito del presidente de la República, Jorge Hernández Campos sostiene que las particularidades del presidencialismo posrevolucionario, se explican en parte porque sus orígenes están inscritos en un pasado de armas. La jerarquía en la que se funda la autoridad de los jefes revolucionarios y de los oficiales funciona con base en principios de disciplina y subordinación. Jorge Hernández Campos, "El fin del mito presidencial” (pp. 47-55), en Enrique Florescano (coord.), Mitos mexicanos, México, Taurus, 2001, p. 51.

12 María Amparo Casar, "Quince años de gobierno sin mayoría en el Congreso mexicano”, Política y Gobierno, vol. xxv, núm. 2, 2013. Véase tam- 
De ahí que, para suplir esta debilidad estructural, los presidentes mexicanos recurrieran a instrumentos metaconstitucionales.

De éstos, el más sobresaliente era el partido hegemónico, el PRI, que fue el soporte fundamental de la presidencia autoritaria. No obstante su identificación como pieza central del régimen autoritario, el PRI mostró una capacidad de supervivencia asombrosa, pues desde 1982, cuando se iniciaron las protestas antiautoritarias a raíz de la nacionalización de la banca y se reorganizaron las fuerzas políticas, pasaron quince años antes de que perdiera la mayoría en la Cámara de Diputados y las gubernaturas. Esta resistencia se explica, en primer lugar, por su vínculo privilegiado con la presidencia de la República y, luego, porque contaba con una estructura organizativa nacional con la que no podían competir las oposiciones.

\section{LAS REFORMAS DE LOS AÑOS NOVENTA Y LA DEBILIDAD DEL ESTADO}

En diciembre de 1982, cuando asumió el poder, el presidente Miguel de la Madrid presentó una propuesta de reforma del Estado que fue el arranque de un proceso que limitaba el intervencionismo estatal, y que se prolongó hasta finales del siglo xx. Una de sus consecuencias fue el debilitamiento de la presidencia, que perdió áreas de competencia e instrumentos de gobierno. Si bien su intención era restablecer los límites del intervencionismo que la nacionalización bancaria había ampliado, ${ }^{13}$ su gobierno es considerado como el iniciador de las reformas liberales. Es cierto que, en 1985, México ingresó al GATT y puso fin a más de cinco décadas de proteccionismo.

A partir de 1989, el presidente Salinas puso en pie un audaz programa de reformas económicas que transformaron el

bién: Will Panstres y Rogelio Hernández Rodríguez, "La democracia mexicana y el retorno del PRI", Foro Internacional, octubre-diciembre de 2012.

${ }^{13}$ Véase Miguel de la Madrid, con la colaboración de Alejandra Lajous, Cambio de rumbo, 1982-1988, México, Fondo de Cultura Económica, 2004, pp. 37-38. 
país. Estas medidas fueron profundizadas por su sucesor, Ernesto Zedillo. El nuevo modelo de crecimiento exigía el abandono del estatismo y la adopción de las ideas, los argumentos, las posturas y las metas de gobierno del Consenso de Washington, que eran la antítesis del nacionalismo revolucionario que había sido la guía del PRI y de los programas de gobierno previos.

El área económica del gobierno se transformó, la política de liberalización modificó el entorno institucional. Era necesario crear reglas y generar procedimientos para hacer realidad la contracción del intervencionismo estatal, la liberalización comercial, la privatización de empresas públicas y la desregulación. Entre 1989 y 2000 se crearon diferentes comisiones con el propósito de regular sectores económicos específicos; asimismo, surgieron órganos de supervisión del cumplimiento de las normas del libre mercado. Se fundaron, por ejemplo, la Comisión Federal de Competencia Económica, Cofeco, y la Comisión Reguladora de Energía, CRE, entre otras.

Las nuevas instituciones asumieron muchas de las funciones que antes desempeñaba el Estado, y desapareció la materia de sus relaciones con algunos actores económicos. Cuando esto ocurrió, el principal agente del crecimiento económico, el presidente, pasó a ser mero testigo de decisiones que en el pasado eran de su competencia exclusiva, por ejemplo, la determinación del monto de la inversión pública, de los precios de ciertos bienes de consumo, o la clase y las cantidades de productos destinados a la exportación. ${ }^{14}$

\section{LA DERRotA DEL PRI}

El factor que de manera decisiva desestabilizó el presidencialismo autoritario fue el fin de la hegemonía del PRI, resultado de un proceso relativamente gradual.

${ }^{14}$ Soledad Loaeza, "La metamorfosis...", art. cit. 
En la sucesión presidencial de 1987, el entonces presidente Miguel de la Madrid no renunció a la costumbre del dedazo, es decir, a la facultad política de designar al candidato presidencial del PRI, lo que equivalía a nombrar a su sucesor en la silla presidencial. No obstante, en esta ocasión, una corriente del partido, encabezada por Cuauhtémoc Cárdenas y Porfirio Muñoz Ledo, reclamó su derecho a participar en la elección del candidato. Su protesta no fue atendida y la escisión de esta corriente, que representaba la izquierda del PRI, se sumó al número creciente de restricciones a la discrecionalidad del presidente que le había sido característica. A partir de ese momento, el partido en el gobierno tuvo que enfrentar el reto de la competencia con otras fuerzas políticas organizadas en partidos.

Para responder a las presiones que demandaban la apertura del proceso sucesorio, el presidente de la Madrid hizo una concesión casi simbólica a la opinión pública y a los priistas descontentos. Los precandidatos -todos miembros de su gabinete- desfilaron en una "pasarela" televisada en la que cada uno de ellos hizo una breve presentación de su diagnóstico del país y de lo que sería su programa de gobierno. Nunca se informaron los resultados de ese ejercicio, pero Carlos Salinas fue el ganador. Aquí lo que me interesa subrayar es que el presidente empezó a responder a las demandas de una opinión pública que en cierta forma ponía un límite a su discrecionalidad.

El presidente Carlos Salinas se propuso llevar a cabo la verdadera transformación de la economía con vistas a su rápida modernización. El país quedó inserto en la globalización. Esta política era profundamente divisiva porque en muchos aspectos era una negación de las políticas estatistas tradicionales, y la fractura del PRI a la que antes aludí está directamente relacionada con la división interna entre reformistas y tradicionalistas. Sin embargo, el partido se disciplinó a la autoridad presidencial y acomodó las decisiones de gobiernos predominantemente tecnocráticos que relegaron a segundo plano los objetivos generales de la tradición revo- 
lucionaria. ${ }^{15}$ Una de las principales razones por las que el descontento se profundizó fue que el PRI pagaba con votos los costos políticos de cambios que no contaban con el apoyo mayoritario de los ciudadanos.

El ánimo reformista era alimentado por el propio presidente Salinas, pero encontró fiera resistencia en el interior de su propio partido. Los priistas no vieron con buenos ojos que, en busca de aliados, el presidente entablara un acuerdo relativamente estable con el PAN, y que reconociera los triunfos de la oposición en las gubernaturas de Baja California, en 1989, y de San Luis Potosí y Guanajuato, en 1991.

El presidente Salinas intentó, aunque no con mucha determinación, reformar el partido; por ejemplo, propuso ajustar los planteamientos "ideológicos" y programáticos al mundo de la globalización. Con ese propósito, buscó sustituir el nacionalismo revolucionario con el liberalismo social. Sus planteamientos quedaron plasmados en la propuesta de una amplia revisión del partido, cuyo objetivo era modernizar la organización anquilosada que no respondía a las transformaciones que había experimentado México. La modernización que perseguía el presidente Salinas demandaba estos cambios, así como la necesidad de responder a las exigencias de una sociedad plural y participativa, cuyas manifestaciones de autonomía empezaron a ganar momentum en coyunturas electorales, primero en el ámbito municipal y, posteriormente, en la disputa por las gubernaturas.

Las tensiones entre la presidencia y el partido no se resolvieron con el ascenso al poder de Ernesto Zedillo. El último presidente del PRI del siglo xx llevó todavía más lejos los cambios que había iniciado su antecesor, a pesar de que esta vía conducía irremediablemente a un enfrentamiento o a una ruptura en la que no habría ganador.

${ }^{15}$ Durante los gobiernos de Carlos Salinas y Ernesto Zedillo, los priistas vivieron la gran incomodidad de tener que apoyar políticas que rechazaban. Sin embargo, en todos los casos se impuso la disciplina de partido. Tiziana Bertaccini y Alberto J. Aguilar Iñarritu, La transformación del PRI. De la oposición a la reconquista del poder, México, Fundación Colosio, 2015. 


\section{LA TERRITORIALIZACIÓN DEL PRI Y LA "SANA DISTANCIA" DE LA PRESIDENCIA DE LA REPÚBLICA}

La reforma del PRI que promovió Salinas aspiraba a ser decisiva para el futuro del partido, pero buena parte de la propuesta que se presentó en la XIV Asamblea, en 1991, fue rechazada por la CTM y por los sectores que manifestaron una firme oposición a los planteamientos presidenciales. Uno de los puntos centrales de la reforma era la adhesión individual al partido, si bien era inaceptable para los sectores, dado que ponía en juego la supervivencia del corporativismo como principio organizativo y la continuidad de las corporaciones como agentes políticos. ${ }^{16}$

La militancia también manifestó su descontento con la arbitrariedad presidencial que había obligado al partido a apoyar candidaturas de tecnócratas, que no habían tenido ningún contacto previo con el PRI o con la política -o había sido esporádico, porque sus carreras se habían desarrollado en la administración pública-, pese a que los priistas habían exigido mayor participación de la militancia en la elección de sus candidatos. ${ }^{17}$ Esta derrota del reformismo interno selló el destino del partido.

El único capítulo de la propuesta que se aceptó fue el relativo a la sustitución de la CNOP por UNE, Ciudadanos en Movimiento, una organización diseñada con base en la territorialización del partido. El cambio tuvo enormes consecuencias porque, al hacer del lugar de residencia el punto básico de organización y ya no el trabajo o el oficio, debilitó a los sindicatos y al PRI, redujo la influencia de las autoridades federales sobre las organizaciones locales, pero favoreció a los gobernadores que rápidamente asumieron una responsabilidad que les permitió controlar municipios y legislado-

${ }^{16}$ Rogelio Hernández, "La reforma interna del PRI", Foro Internacional, vol. xxxiı, núm. 2 (1991), pp. 223-249.

${ }^{17}$ Loc. cit. 
res locales. ${ }^{18}$ Este poder fue el basamento de la construcción que encumbró a Enrique Peña Nieto como el gran componedor del PRI.

Sin embargo, el verdadero final de la hegemonía priista ocurrió en el segundo trienio del mandato de Ernesto Zedillo, el tecnócrata por antonomasia. ${ }^{19}$ En las elecciones federales de 1997, el partido perdió la mayoría en la Cámara de Diputados, mientras que las oposiciones sumadas alcanzaban la mayoría.

La explicación inmediata de esta derrota era la mala relación del PRI con el presidente Zedillo quien, desde el día que tomó posesión, renunció a dirigir el partido para igualar el terreno de la competencia electoral. Anunció su decisión con la promesa -o advertencia- de que entre el partido y el gobierno habría "una sana distancia", lo que equivalía a decir que cortaba el lazo umbilical que unía al PRI con la presidencia de la República. ${ }^{20}$ A partir de entonces se redujeron sustancialmente las ventajas y los privilegios, derivados de recursos públicos, que habían sostenido la hegemonía del PRI durante décadas.

La integración del gabinete del presidente Zedillo fue la confirmación de la desconfianza que le inspiraban los priistas, cuando designó procurador general de la República a Antonio Lozano Gracia, que era un miembro destacado del PAN. Su primer encargo fue la investigación de los crímenes políticos que segaron la vida del candidato del PRI, Luis Donaldo

${ }^{18}$ Guadalupe Pacheco Méndez, "El PRI, relación interna de fuerzas y conflictos en la víspera del proceso electoral de 2006”, Política y Gobierno, vol. XVI, núm. 1 (2009), pp. 157-190.

19 "Zedillo era un tecnócrata del gabinete de Salinas; un tipo gris, sin ninguna militancia en el partido, un tecnócrata, claramente los nuevos cuadros del neoliberalismo. Era un candidato muy rígido [...] pero gracias a la estructura del partido, a la popularidad del presidente, al sentimentalismo de la gente que nos habían matado a nuestro candidato, tuvimos una campaña exitosa y ganamos", Bertaccini y Aguilar, op. cit. (entrevista con Samuel Aguilar, 13 de septiembre de 2013).

${ }^{20}$ Rogelio Hernández Rodríguez, "Ernesto Zedillo, la presidencia contenida", en Foro Internacional, vol. xLIII, núm. 1 (2003), pp. 39-70. 
Colosio, en marzo de 1994, y del secretario general del PRI, José Francisco Ruiz Massieu, en agosto de ese mismo año. Zedillo explicaba su decisión con el argumento de que era una manera de garantizar la imparcialidad de la investigación.

La desconfianza que denotaba esta designación envenenó las relaciones entre el PRI y el presidente de la República, y la distancia propuesta se convirtió en franca hostilidad entre un partido que no se reformaba ni a la velocidad ni en el sentido que el presidente hubiera deseado, y la misma organización que buscaba sacudirse el yugo presidencial.

La promesa de autonomía era en sí misma un reto para el PRI, que tendría que encontrar recursos políticos y económicos para sostenerse sin el apoyo que hasta entonces le garantizaba la presidencia. Aunque en realidad el presidente nunca hizo una exposición detallada de qué entendía por la reforma del PRI, es probable que pensara que la escasez de recursos sería el detonador de una reforma de gran calado.

El efecto de corto plazo de la disminución de los privilegios del PRI ${ }^{21}$ fue reforzar el control que los gobernadores ejercían sobre los priistas de su entidad, pues se apresuraron a llenar el vacío que dejaba la renuncia del presidente a asumir el liderazgo del partido, para impulsar sus intereses de manera egoísta. Esta evolución fue una advertencia de las consecuencias desastrosas que tendrían, primero, la derrota de 2000 y, doce años después, la victoria.

${ }^{21}$ Para los priistas, el corte del cordón umbilical del partido y la presidencia de la República fue una decisión imperdonable que generó entre ellos un resentimiento y un rencor insuperables hacia Ernesto Zedillo. En una entrevista que dio en septiembre de 2013, Manuel Bartlett afirmó rabioso: "El PRI de Salinas es Salinas. Zedillo es otra mistificación. Zedillo ni fue político ni fue nada, Zedillo era un mediocre que le cargaba el portafolio a Aspe cuando llegaban a verme a mí en Gobernación. Zedillo es producto del asesinato de Colosio, y la creación de un personaje que nunca pensó que podía hacer nada... Zedillo a mí me decía, yo era gobernador de Puebla, el PRI es una porquería, quítate de estas cosas ¿A mí me lo decía!. El PRI no sirve”, citado en Bertaccini y Aguilar, op.cit., p. 175 . 
Cortar el cordón umbilical que vinculaba al PRI con la presidencia de la República, significaba perder el apoyo financiero y de otro tipo que el partido recibía del gobierno; pero, al mismo tiempo, el presidente exigía el apoyo de los legisladores priistas a medidas impopulares que le costaban votos al partido, un costo del que el presidente Zedillo nunca se hizo cargo; así ocurrió con el agresivo recorte presupuestal hecho para enfrentar la severa crisis financiera de 1994, o el incremento de 50\% al IVA. Los resultados electorales del PRI reflejaron el impacto de estas medidas sobre el ánimo de la opinión pública.

La rotación de presidentes del partido es un claro indicador de la desestabilización que sufrió el PRI en estos años. Durante la presidencia de Carlos Salinas, el partido tuvo cinco presidentes; con Zedillo el número aumentó a seis, y a siete en tiempos del presidente Peña Nieto; entre 1982 y 2018, tuvo 26 presidentes. ${ }^{22}$

El debilitamiento del PRI trajo consigo el de la presidencia. Además, la erosión del poder presidencial que implicaban estos cambios, se acentuó gracias al surgimiento de nuevas formaciones partidistas: el Partido Verde Ecologista de México, PVEM, y el Partido del Trabajo, PT, y al fortalecimiento de los partidos de oposición. En 1997, por primera vez en la historia, el partido del presidente no alcanzó una mayoría absoluta en la Cámara de Diputados y se vio obligado a negociar el apoyo de otras fuerzas políticas. Esta situación de gobierno dividido debilitaba a esas instituciones, y se repitió en las presidencias de Vicente Fox y Felipe Calderón, que tuvieron que trabajar con legislaturas en las que el PAN era minoritario. ${ }^{23}$

22 Nada más entre 2012 y 2019 fueron siete. Cristina Díaz Salazar (30 de noviembre de 2012 a 11 de diciembre de 2012); César Camacho (11 de diciembre de 2012 a 20 de agosto de 2015); Manlio Fabio Beltrones (20 de agosto de 2015 a 20 de junio de 2016); Carolina Monroy del Mazo (21 de junio de 2016 a 12 de julio de 2016); Enrique Ochoa (13 de julio de 2016 a 2 de mayo de 18); René Juárez (mayo de 18 a 16 de julio de 2018); Claudia Ruiz Massieu (16 de julio de 2018 a 18 de agosto de 2019).

${ }^{23}$ Véase Casar, art. cit. 
La pluralización de la representación en el Poder Legislativo se afianzó como un rasgo distintivo de la democracia mexicana, sujeto a las restricciones que le imponía la ausencia de mayorías en el Congreso.

La transformación del PRI, de haber sido una organización relativamente monolítica a convertirse en un conjunto desordenado y diverso de organizaciones dispares, repercutió en el Poder Legislativo que también se libró del control presidencial. No obstante, a partir de esos cambios, los resultados electorales del partido mejoraron de manera significativa.

En 2009, el PRI registró un impresionante resurgimiento: de 16 gubernaturas en juego ese año, triunfó en 15; y en las elecciones federales fue el partido ganador: se convirtió en la primera fuerza en el Congreso; el grupo parlamentario priista fue el más importante de la LXI Legislatura, con 184 diputados de mayoría relativa y 53 de representación proporcional. ${ }^{24}$

El análisis detallado de esta evolución probablemente destacaría, en primer lugar, la fuerza de los gobernadores; luego, el enorme gasto en publicidad de los gobiernos estatales, así como un apoyo casi ilimitado a las representaciones locales del PRI. Varios de estos gobernadores habían recibido apoyos extraordinarios para sus campañas electorales, del entonces gobernador del Estado de México.

Peña Nieto creó dos organizaciones que fueron el instrumento para movilizar apoyos y ganar lealtades personales a espaldas del PRI: Fuerza Mexiquense, que actuó en el Estado de México durante su responsabilidad como gobernador, y Expresión Política Mexiquense, que se instaló en el ámbito nacional y llegó a manejar 90000 operadores en todo el país, con el fin de apoyar la elección de los siguientes gobernadores: Fernando Toranzo, de San Luis Potosí, Yvonne Ortega, de Yucatán, José Francisco Olvera, en Hidalgo, Javier Duarte, de Veracruz, y Jesús Calzada, en Querétaro. Estos apoyos se traducían en el

24 Rosa María Mirón Lince, "Recomposición del PRI en la primera década del milenio", Convergencia, vol. 19, núm.55 (mayo-agosto de 2012), pp. 43-67. 
compromiso de apoyar a Peña Nieto en la competencia interna del PRI por la candidatura presidencial ${ }^{25} \mathrm{y}$, más adelante, en su campaña presidencial.

\section{EL ASCENSO DE LOS GOBERNADORES}

Los extraordinarios resultados de 2009 hicieron olvidar al PRI la debilidad con la que había llegado a la elección de 2000, herido de muerte por la cuasi ruptura con la presidencia de la República, dividido por las diferencias entre grupos rivales, y desorientado por el cambio de modelo económico y por el reformismo político que le había arrebatado sus privilegios y lo obligaba a competir en condiciones de incertidumbre. La ausencia de un liderazgo unificado que fijara las reglas de la competencia interna, las líneas generales de acción del partido y un programa común también tuvieron un costo muy alto para el partido en cuanto a coherencia y eficacia electoral.

La renuncia del presidente Zedillo a ejercer el liderazgo del PRI decapitó al partido, propició las divisiones internas, el faccionalismo y, sobre todo, la balcanización del partido. Los beneficiarios de esta descomposición fueron los gobernadores, que asumieron la dirección partidaria en sus respectivos estados, ${ }^{26}$ y los legisladores en el Congreso transfirieron su lealtad y su disciplina del presidente de la República al gobernador, que obtuvo una autonomía que impuso una novedosa restricción al poder presidencial.

A partir de esa ampliación de su poder, los ejecutivos estatales desarrollaron una capacidad de negociación con el gobierno federal que les era hasta cierto punto desconocida. ${ }^{27}$

25 Arturo Rodríguez García, "Populismo en plástico", Proceso, núm. 1810, 26 de junio 2011.

${ }^{26}$ Pacheco Méndez, art. cit.

${ }^{27}$ Los primeros ejemplos de este fortalecimiento de la autonomía de los gobernadores se presentaron en San Luis Potosí y en Guanajuato, donde el presidente Salinas tuvo que aceptar las exigencias locales y sus reclamos contra los resultados oficiales de las elecciones que habían declarado 
Estos cambios se vieron favorecidos por una reforma fiscal que aumentó considerablemente los ingresos de los gobiernos estatales, pues reglamentaba la entrega y distribución de recursos federales que correspondían a cada uno de los estados. En 1998 se votó una ley que multiplicó las aportaciones que recibían del gobierno federal, pero las recibieron antes de que se diseñaran mecanismos de fiscalización que controlaran de alguna manera a gobernadores que nunca antes tuvieron autonomía fiscal. ${ }^{28}$

Los gobernadores no sólo ganaron autonomía en relación con el presidente de la República, sino que su poder en el propio estado se acrecentó, dado que podían tomar decisionesindependientes en relación a munícipes ylegisladores, ${ }^{29}$ y muchos empezaron a comportarse como "virreyes". ${ }^{30} \mathrm{El}$ presidente, por su parte, no tenía muchos instrumentos para controlarlos. En su propia defensa, los gobernadores apelaban, en primera instancia, al federalismo y a la soberanía estatal; luego evocaban el compromiso con la descentralización.

No obstante lo anterior, la fuerza que hubieran podido desarrollar los gobernadores se veía relativizada por la hipoteca que representaban las organizaciones criminales, y la violencia que se extendía por buena parte del territorio na-

victoriosos a los candidatos priistas, Fausto Zapata Loredo y Ramón Aguirre, respectivamente. En ambos casos la oposición, en este caso dirigida por el PAN, hizo valer la fuerza que había mostrado en la elección presidencial de 1988, en la que había asegurado más de cien diputaciones.

28 Juan Carlos Moreno Bagatella, "Equilibrios de poder en las entidades federativas. Estudio longitudinal de las legislaturas locales en México en el periodo 2000-2014", Revista Mexicana de Análisis Político y Administración Pública, vol. III, núm. 2 (2014), pp.125-154.

29 "Los gobernadores contaron con las mismas facultades metaconstitucionales del presidente en sus jurisdicciones, eran los jefes reales del partido, podían nombrar a los candidatos a las presidencias municipales y diputaciones locales...", loc. cit. y Andrew Paxman (coord.), Los gobernadores, caciques del pasado y del presente, México, Grijalbo, 2018.

30 Véase, por ejemplo, Daniela Pastrana, "Javier Duarte (Veracruz, PRI, 2010-2016). Un corazón muy chiquito", en Revista Mexicana..., op. cit., pp. 271-306. 
cional. Entre 2011 y 2018 los homicidios dolosos aumentaron de más de 27000 a 36000 .

La política de descentralización, que fue una de las reformas más importantes de los gobiernos anteriores, aplicada primero por Carlos Salinas y que todos sus sucesores mantuvieron, también contribuyó a este gran proceso de desmantelamiento del Estado autoritario.

Los presidentes panistas apoyaron con entusiasmo la política de descentralización, que respondía a su vieja protesta contra la centralización y a su demanda de respeto al municipio libre y a la soberanía de los estados, de manera que profundizaron su alcance. Los gobernadores, fortalecidos de esta manera, se desentendieron cada vez más del poder federal y de sus objetivos. La autonomía en el manejo de los recursos públicos se prestó a las peores prácticas de enriquecimiento ilícito; en 2017, el periódico estadounidense The New York Times reportó que 17 exgobernadores mexicanos eran sospechosos de haber incurrido en corrupción y estaban siendo investigados. ${ }^{31}$

El proceso judicial del exgobernador de Veracruz, Javier Duarte, es ilustrativo de esta perversión. Fue encarcelado en 2017 acusado de desviar cerca de 4500 millones de pesos del erario, en perjuicio sobre todo de programas sociales. ${ }^{32}$ Además, durante el gobierno de Duarte, Veracruz fue el estado que registró mayor número de periodistas asesinados. La estrategia del gobernador era la misma que la de Ángel Aguirre en Guerrero, minimizar su importancia o culpar a las víctimas de supuestos delitos que, desde su punto de vista, justificaban el crimen. ${ }^{33}$ El gobierno federal cerró los ojos a los abusos de este gobernador que había apoyado la candidatura de Peña Nieto en la competencia interna, luego de los recursos que él había recibido del gobernador del Estado de México para su campaña por la gubernatura.

31 Paxman, Los gobernadores, op.cit., p. 11.

32 Pastrana, art. cit.

33 Claudio Lomnitz, “¿Qué sucede en Veracruz?”, La Jornada, 17 de junio de 2015 . 


\section{LA PERSONALIZACIÓN DEL PODER PRESIDENCIAL}

Muchos son los autores que privilegian el papel del personaje que ocupa la presidencia en el estudio del régimen presidencial. No obstante, en condiciones normales, las circunstancias en las que gobierna un presidente y los instrumentos que tiene para hacerlo se imponen a sus rasgos de personalidad, y ésta pierde relevancia analítica frente a factores estructurales, por ejemplo, la posición geopolítica del país en cuestión, las circunstancias o los recursos formales e informales que se atribuyen a los presidentes.

La personalización del poder político es un fenómeno recurrente y, ya no como derivación exclusiva del régimen presidencial, se ha extendido y fortalecido, ha afectado incluso a los regímenes parlamentarios, donde el primer ministro se ha convertido en una figura central que concentra los recursos de la fuerza política que representa, atrae toda la atención de la opinión pública que, más que a su partido, responsabiliza a él o a ella, en lo personal, de las acciones del gobierno. ${ }^{34}$ Así, por ejemplo, los medios dan el mismo tratamiento a la canciller alemana Angela Merkel, a las declaraciones del primer ministro británico, Boris Johnson, o del presidente de gobierno español, Pedro Sánchez, que recibe el jefe del Ejecutivo de un régimen presidencial. Este desarrollo no es sorprendente, a pesar de que en estos regímenes el poder no lo ejerce sólo una persona y de que el primer ministro fue elegido como parte de una fuerza política, y no como individuo.

La inclinación a ver en los rasgos y las características personales del presidente la fuente del poder que ejerce se ha acentuado recientemente como consecuencia del papel decisivo de la televisión y ahora las redes sociales en la comunicación política, porque tienden a privilegiar la imagen individual, ilustran la información concentrando la mirada en la persona

${ }^{34}$ Véase, por ejemplo, José Luis Cheibub, Zachary Elkins y Tom Ginsburg, "Beyond presidentialism and parliamentarism", British Journal of Political Science, vol. 44, núm. 3 (julio de 2014), pp. 515-584. 
del jefe de gobierno. Además, el estilo estridente y el tono superlativo propios de la prensa del espectáculo y del corazón, que construyen personajes a partir del aspecto físico y de sucesos de la vida privada, se ha impuesto a la información relativa a los políticos, de tal manera que se ha producido una confusión entre el hombre de Estado, el líder político y la "celebridad". Enrique Peña Nieto y sus estrategas cultivaron esta confusión que podía justificarse en una campaña electoral, pero la prolongaron al ejercicio de la presidencia de la República. Perdieron de vista que, como lo observa Richard Rose, "Lo que se necesita para convertirse en presidente no tiene nada que ver con lo que se necesita para ser presidente". ${ }^{35}$

En la elección presidencial de 2012, el candidato del PRI, Enrique Peña Nieto, triunfó con 39 por ciento del voto frente a la candidata del pan, Josefina Vázquez Mota, que recibió 26 por ciento, y el candidato de la Coalición por el Bien de Todos, Andrés Manuel López Obrador, aceptó los resultados oficiales que le acreditaron 32 por ciento de sufragios.

Hay por lo menos tres posibles explicaciones del regreso, hasta cierto punto inesperado, del PRI: ganó la elección gracias al candidato, ${ }^{36}$ a la transformación del partido ${ }^{37} \mathrm{o}$ a la impopularidad y la desilusión que causó la incompetencia de dos gobiernos panistas. ${ }^{38} \mathrm{Si}$ aceptamos la primera de ellas entonces hay que concentrarse en la personalidad de Enrique Peña Nieto.

35 Richard Rose, citado en Mark A. Peterson, "Presidential power and the potential for leadership" (pp. 363-379), en Robert Y. Shapiro, Martha Joynt Kumar y Lawrence R. Jacobs (eds.), Presidential Power. Forging the Presidency of the XXIst Century, Nueva York, Columbia University Press, 2003 , p.369.

36 Carlos Tello Díaz, "La senda del rockstar", Nexos, junio de 2012.

37 Véase, por ejemplo, Rogelio Hernández y Will C. Panters, "La democracia en México y el retorno del PRI", Foro Internacional, núm. 52 (2012), pp. 755-795.

38 Gilles Serra, "Demise and resurrection of a dominant party: Understanding the PRI's Comeback in Mexico", Journal of Politics in Latin America 5, núm. 3 (diciembre de 2013), pp. 133-154. 
Su candidatura fue apadrinada por un poderoso político del Estado de México, Arturo Montiel, él mismo aspirante a la presidencia del República, pero tuvo que renunciar a sus ambiciones por las muchas acusaciones de corrupción en su contra. A Peña Nieto también se le atribuye el apoyo del llamado "Grupo Atlacomulco”. Su existencia es materia de discusión pero, en caso de ser real, estaría formado por políticos profesionales originarios de esa localidad, vinculados además por lazos familiares, de amistad o profesionales, que constituyen un manto de protección, una red de apoyo y un trampolín para los suyos. Se afirma que este grupo fue fundado por Isidro Fabela y han pertenecido a él no menos de ocho gobernadores, tres de los cuales integran ya una dinastía: Alfredo del Mazo Vélez, Alfredo del Mazo González y Alfredo del Mazo Maza. ${ }^{39}$

En 2005, Peña Nieto fue elegido gobernador de un estado que representaba el 13 por ciento de la población nacional y el 9 por ciento del PIB. Desde entonces empezó a hablarse de él como un hábil político, epítome de una nueva generación de priistas y una promesa para la presidencia de la República. La imagen que de él se promovía era la de un político eficaz que cumplía lo que prometía.

Desde entonces Peña Nieto estableció una relación estrecha con Televisa, que vio en él a un político telegénico cuya carrera podía promover y así "capturar" al futuro presidente de la República. Éste fue el objetivo de la televisora que se comprometió a fondo con la cobertura de la campaña presidencial del candidato del PRI, a quien además asesoró para convertirlo en un rockstarirresistible. En entrevistas con funcionarios de Televisa, Carlos Tello Díaz recogió afirmaciones como ésta: se trataba de "vender no al partido, que era invendible, ni las propuestas del candidato que nadie recordaría, sino al personaje Peña Nieto". ${ }^{40}$ Había que

39 Álvaro Arreola, "Isidro Fabela y Alfredo del Mazo Vélez (Estado de México, PRM/PRI, 1942-1951” (pp. 31-59), en Paxman, op. cit.

40 Tello Díaz, art. cit. 
"vender un rockstar apuesto, joven, alegre, cálido, con muy buenas formas". 41

Televisa se enriqueció gracias a una campaña política que fue enteramente financiada con recursos públicos que llegaron a raudales a los bolsillos de los dueños de las televisoras. ${ }^{42}$ El éxito obtenido en la elección hizo creer al PRI, a Televisa y al propio Peña Nieto que sus atributos personales bastaban para gobernar un país que estaba devastado por una guerra inacabable contra el crimen organizado, por el descontento que causaba la incapacidad del presidente Felipe Calderón de sacar adelante reformas estructurales que las oposiciones bloqueaban en el Congreso, por la expansión de la violencia criminal, la creciente desigualdad, el desempleo y la falta de crecimiento económico. Por último, el comportamiento de Peña Nieto en la presidencia de la República sugiere que tanto él como su gabinete pretendieron gobernar como si nada hubiera cambiado desde el fin del último gobierno priista, como si su elección hubiera sido por sí sola una restauración.

\section{LA PRESIDENCIA ARRINCONADA}

Enrique Peña Nieto recibió de Felipe Calderón una presidencia disminuida por el desarrollo de la partidocracia, el fortalecimiento del Poder Legislativo, la autonomía de los gobernadores, la independencia de los legisladores, las reformas económicas y los órganos autónomos.

Para gobernar, el nuevo presidente necesitaba redimensionar la institución, crear nuevos anclajes institucionales o renovar los existentes, pues ni siquiera sabía con certeza si el PRI le sería útil para la reconstrucción. Sin embargo, el recur-

${ }^{41}$ Loc. cit.

42 Andrew Paxman, "Televisa de 1997 a 2018: el socio fallido de la democratización”, en José Galindo (coord.), México contemporáneo. Aspectos económicos, políticos y sociales, Xalapa, Universidad Veracruzana, 2018. 
so más expedito que tuvo a la mano Peña Nieto fue él mismo, y apostó su capacidad de gobierno a sus atributos personales, los verdaderos y los que le sumó la imagen que crearon sus publicistas, que lo presentaron no sólo como una cara bonita, sino como un político disciplinado, ordenado y cumplidor. El lema central de su campaña fue el mismo que había utilizado en la campaña para la gubernatura del Estado de México: "Te lo prometo, te lo firmo y te lo cumplo”.

Había, sin embargo, grandes dudas en torno al gobernador Peña Nieto. Se le acusaba de haber emprendido "obras faraónicas”, y los contratos de obra se habían mantenido en la oscuridad, pero se sabía que había empresas favorecidas por su gobierno. ${ }^{43}$ Por ejemplo, la empresa española oHL y el grupo de empresas Higa habían recibido en forma consistente los contratos más importantes para la construcción de grandes obras de infraestructura.

$\mathrm{Al}$ mismo tiempo que se levantaban edificios de lujo, los índices de pobreza y desempleo en el Estado de México eran superiores a la media nacional, y los feminicidios habían aumentado durante la gubernatura de Peña Nieto. ${ }^{44}$ Peor todavía, su mentor y predecesor, Arturo Montiel, había sido acusado de graves actos de corrupción, pese a lo cual nunca fue investigado.

El primer paso que dio el presidente Enrique Peña Nieto para construir su liderazgo a nivel nacional fue convocar a todas las fuerzas políticas representadas en el Congreso a debatir y, de ser posible, negociar la aprobación de las reformas

${ }^{43}$ Es el caso de las empresas que forman el Grupo Higa, propiedad de Juan Armando Hinojosa, que recibió muchos contratos, primero, en el Estado de México, y luego en obras de importancia nacional, como el tren rápido México-Querétaro. Este grupo fue objeto de una investigación de la Cámara de Diputados en 2015. Véase Lidia Pérez Bárcenas, "Higa: una historia de corrupción e impunidad", en Morena, Regalos obscenos. Lo que no pudo esconder el Pacto contra México, México, Cámara de Diputados LXXII Legislatura, 2015, pp. 83-134.

${ }^{44}$ Jenaro Villamil, "Meganegocios, relumbrón, quimera, pobreza", Proceso, 3 de julio de 2011, proceso.com.mx 
estructurales que estaban pendientes desde 2000. La ausencia de una mayoría presidencial en el Legislativo era una de las explicaciones más socorridas de esta casi parálisis del Congreso, y se atribuía a las malas relaciones del presidente Calderón con su partido y con el Poder Legislativo.

El presidente Peña Nieto logró sentar a la mesa de discusión a las tres principales fuerzas políticas del país que hasta entonces se miraban con desconfianza y habían sido incapaces de entablar un diálogo constructivo. Esta reconciliación fue en buena medida obra del presidente que adoptó un lenguaje moderado e incluyente. El acuerdo entre el PRI, el PAN y el PRD, inyectó optimismo en el ambiente y generó una atmósfera de entusiasmo. Una ola de admiración recorrió a la opinión pública que estaba hastiada de los jaloneos del pasado entre el presidente y los partidos o los legisladores, de lo que consideraba la incapacidad para dialogar de los actores políticos.

El Pacto por México consistía en cinco grandes acuerdos cuyo objetivo era crear una sociedad de derechos y libertades, fomentar el crecimiento económico, el empleo y la competitividad, la seguridad y la justicia, la transparencia y la rendición de cuentas, y el combate a la corrupción. Entre 2012 y 2014 las relaciones en el seno del poder legislativo transcurrieron sin grandes conflictos, y las reformas fueron negociadas, votadas y aprobadas. ${ }^{45}$ No obstante, el debate dividió a las fuerzas políticas, agravando la fragmentación; es decir, no lograron el consenso que había pretendido el presidente, tan es así que, por ejemplo, el Sindicato Nacional de Trabajadores de la Educación, SNTE, no pudo frenar a la disidencia ni disolver las tensiones internas que provocaba la reforma educativa. El PRD también fue víctima de este impacto divisivo y se escindió; así nació el Movimiento de Regeneración Nacional, Morena, fundado por Andrés Manuel López Obrador.

${ }^{45}$ Miguel Armando López Leyva, "El episodio reformista en México (2012-2014). Explorando las razones del cambio en la alternancia”. Estudios Políticos, $9^{\text {a }}$ época, núm. 35 (mayo-agosto 2015), pp. 61-85. 
Más allá de sus virtudes inmediatas, el Pacto planteaba un problema de consideración: era un acuerdo que se había concluido al margen del Congreso, un arreglo extraparlamentario cuya base era la igualdad ficticia de todos los participantes. Sometida la discusión de las reformas estructurales a la mecánica parlamentaria, habrían surgido las diferencias en la representación de las fuerzas políticas, y la necesidad de negociar alianzas y apoyos. El PRI no tenía mayoría y, desde el punto de vista táctico, le convenía sustraer el debate del Congreso porque si todas las fuerzas políticas eran iguales, la negociación entre ellas sería más fácil y menos costosa. La gran desventaja de semejante estrategia fue que relegó a segundo plano la institución clave de la representación nacional. En lugar de fortalecer a la institución presidencial, esta estrategia se atribuía exclusivamente a la personalidad del presidente y a su habilidad política.

De todas estas reformas, la más efectiva fue la reforma hacendaria que se presentó a las cámaras en septiembre de 2013, cuyo decreto firmó el presidente en enero del año siguiente. Su objetivo era aumentar la captación de recursos públicos, asegurando el pago de impuestos de empresas grandes y medianas, y eliminando regímenes especiales. El gobierno esperaba recaudar alrededor de 18000 millones de dólares adicionales, o sea 1.4\% del PIB; gracias a esta reforma, los ingresos tributarios del gobierno federal pasaron de 1157913 millones de pesos en 2012, a 2150172 millones de pesos en 2018.

La continuidad de la política económica mantuvo a niveles mediocres el crecimiento, aunque se controló la inflación, pero se crearon pocos empleos. No obstante, el naufragio del gobierno de Peña Nieto puede atribuirse más que a la economía, a la pareja letal que formaron la violencia y la corrupción.

\section{Corrupción Y Violencia}

Desde hace ya varios años uno de los temas de mayor preocupación para los mexicanos ha sido el creciente poder de 
bandas de narcotraficantes que se han extendido en casi todo el territorio nacional. Se han aplicado diferentes políticas de seguridad para atacar el problema: las entidades gubernamentales responsables de investigar, perseguir, detener y castigar a los delincuentes se han reorganizado varias veces, pero el poder de las organizaciones criminales sólo se ha extendido, y ninguna de esas políticas de seguridad ha funcionado.

Durante el gobierno del presidente Felipe Calderón, el ejército asumió la responsabilidad del combate al narcotráfico. El programa consistía principalmente en atacar a las cabezas de las organizaciones. Esta estrategia contaminó al ejército, pero además provocó la fragmentación de las bandas y, por ende, la multiplicación de estos grupos. El tamaño y el poder que han alcanzado son un desafío para la soberanía del país. Sus recursos financieros y de armamento son muy superiores a los de la policía, el ejército y la armada.

Tal vez el peor episodio de la presidencia de Enrique Peña Nieto, el más doloroso y también el más revelador de la debilidad del Estado, fue la tragedia de Ayotzinapa, que demostró la insidiosa penetración del narcotráfico en las instituciones mexicanas: policías locales, centros educativos y el ejército. En la madrugada del 26 al 27 de septiembre de 2014, desaparecieron nueve jóvenes y 43 estudiantes de la Normal Rural de Ayotzinapa, que fueron asesinados e incinerados presuntamente por policías municipales de Iguala.

Ante la magnitud del crimen, el presidente desconcertado reaccionó como si careciera de instrumentos para combatir y castigar a los responsables. Peña Nieto, el secretario de Gobernación, Miguel Ángel Osorio Chong, el Procurador General de la República, Jesús Murillo Karam, y el secretario de Educación, Aurelio Nuño, dejaron la solución de la crisis en manos del gobernador de Guerrero, Ángel Aguirre. Éste se mostró "pasivo", y no tuvo ni la capacidad ni la disposición para investigar lo ocurrido en Ayotzinapa -una ineficacia que la opinión pública le reprochó al Ejecutivo federal, antes que al gobernador. 
La aparente pasividad del presidente Peña Nieto quizá se explique por el involucramiento de priistas en la tragedia; o simplemente porque las dimensiones del problema eran tales, que lo intimidaron. La investigación descubrió que la orden de "desaparecer" a los 43 estudiantes la había dado José Luis Abarca, presidente municipal de Iguala. ${ }^{46}$

Ayotzinapa también mostró que la flamante autonomía de los gobernadores se topaba, en primer lugar, con el poder de organizaciones criminales que ponen en riesgo la continuidad del Estado mexicano. Cuatro meses después de los acontecimientos de Ayotzinapa, el procurador Murillo Karam ofreció una explicación del crimen. Esta última versión de lo ocurrido sostiene que se trató de una confusión de las policías municipales que pensaron que los muchachos no eran estudiantes, sino que se trataba de narcotraficantes. Ésta es la verdad histórica" que nunca ha sido aceptada. ${ }^{47}$

Ayotzinapa es emblemático del desorden y las confusiones que ha provocado el combate al narcotráfico entre el gobierno federal, los gobiernos estatales y municipales. También ilustra la decadencia de una clase política que, ayuna de ideas y comprometida solamente con una imagen -la modernidad-, en su empeño por sustraerse a las reglas y beneficiarse personalmente de los recursos públicos, se autodestruyó.

Después de 2015 afloraron escándalos de corrupción casi todos los meses ${ }^{48}$ el mayor de ellos fue la, así denominada, "Estafa maestra". Una investigación de Animal Político y la or-

${ }^{46}$ Roberto Rock L., La historia detrás del desastre. Crónica de una herencia envenenada, México, Grijalbo, 2019, pp. 98-99.

${ }^{47}$ La investigación dio lugar a que se mirara con cuidado la trayectoria del gobenador. El procurador Murillo Karam había integrado un expediente de Ángel Aguirre, quien estaba involucrado en varios homicidios y en negocios que se habían beneficiado de recursos públicos, y la Comisión de Derechos Humanos lo responsabilizaba de la muerte de tres personas en Chilpancingo, Guerrero. Aguirre jamás pisó la cárcel; se mantuvo a salvo bajo el manto protector de Osorio Chong, su aliado. Véase ibid, p.100.

${ }^{48}$ En 2015, Transparencia Internacional dio a México una calificación de 3.1 en materia de corrupción. 
ganización civil Mexicanos contra la Corrupción y la Impunidad (MCGI) que reveló un desvío de recursos públicos de más de 7000 millones de pesos a través de operaciones trianguladas en las que intervinieron once dependencias del gobierno federal, varias universidades y empresas fantasma. La Secretaría de Desarrollo Social, cuya titular era Rosario Robles, fue la protagonista más activa de este programa. Las investigaciones al respecto están en manos del gobierno lopezobradorista.

\section{EL PRI: LA ÚLTIMA OPORTUNIDAD}

Los resultados de las elecciones federales de 2015 no fueron buenos para el PRI, pero tampoco catastróficos. El partido obtuvo 29 por ciento de la representación, es decir, perdió nueve puntos porcentuales respecto a lo obtenido tres años antes, de manera que fue mayor la necesidad de tener aliados en el Congreso; gracias al PVEM alcanzaba 36 por ciento, mientras que el PAN, su competidor más cercano, en solitario registró 26 por ciento del voto, y Morena se estrenó en la Cámara de Diputados con una bancada de 35 legisladores.

Estos resultados estabilizaron al PRI pero, en noviembre de 2015, una investigación periodística reveló que el presidente Peña Nieto y su esposa, la actriz de telenovelas, Angélica Rivera, habían adquirido una mansión de un valor de 7 millones de dólares en las Lomas de Chapultepec. La operación de compra-venta planteaba un conflicto de interés, porque había sido diseñada y construida por uno de los contratistas más favorecidos por el gobierno de Peña Nieto en la asignación de contratos de obra pública. ${ }^{49}$

La información sobre la Casa Blanca tomó proporciones mayúsculas y el presidente dio marcha atrás y anuló la compra de la casa -para disgusto de su propia esposa-, pidió per-

${ }^{49}$ Mauricio Merino, "El precio de la Casa Blanca”, en Morena, op.cit., pp. 155-157. 
dón a los mexicanos por la insensibilidad que lo había llevado a ostentar gran riqueza a los ojos de una población que tenía graves dificultades económicas o que vivía en pobreza extrema. El escándalo se acalló cuando se anunció que la compra de la casa se había suspendido. Este incidente ejemplifica los riesgos que acarrea la confusión del mundo de las celebridades con el mundo de los políticos; su ética, sus normas de comportamiento, sus objetivos son radicalmente distintos. Sin embargo, el daño estaba hecho.

La opinión pública fue un juez severo de Enrique Peña. Su gobierno terminó con una imagen negativa de corrupción, pasividad e ineficiencia y, según declaró en televisión Alejandro Moreno, director de encuestas de El Financiero, el 27 de noviembre de 2018 había un nivel de aprobación de 26 por ciento, sin precedentes, en tanto que su sucesor, Andrés Manuel López Obrador llegaba a la presidencia con 66 por ciento de aprobación.

\section{Conclusiones}

El periodo presidencial 2012-2018 pasará a la historia como el más corrupto y el más frívolo de la historia del PRI pero, antes de hacer un juicio sumario de estos años, habrá que preguntarse cuáles fueron las condiciones institucionales que propiciaron los comportamientos francamente delictuosos de más de un funcionario público de alto nivel y de varios políticos. También habrá que reflexionar sobre las consecuencias no deseadas que tuvieron las reformas liberales sobre el Poder Ejecutivo.

La fragmentación de las fuerzas políticas no acarreó la aparición de liderazgos nuevos. El presidente Peña Nieto no tenía los instrumentos para construirlo y quedó inerme frente a la ofensiva de los grandes intereses privados.

El desmantelamiento del Estado intervencionista se concentró en la dimensión económica, mientras que el desarrollo de instituciones y actores políticos quedó a la deri- 
va. Esta situación indica que el presidencialismo mexicano había perdido capacidad de liderazgo, y que fracasó cuando para recuperarse recurrió al frágil y voluble apoyo de la personalización del poder. Este fenómeno ha sido explicado como un producto de la historia y/o de la cultura. Sin embargo, también ha sido una estrategia de gobierno, el instrumento de actores políticos que han querido apoyarse en esos factores para construir un liderazgo plebiscitario con poderes ampliados. La experiencia de Enrique Peña Nieto -y de Vicente Fox, que también lo intentó-, es prueba de que el poder del presidente, la trascendencia de sus decisiones después de su sexenio necesita mucho más que historia y cultura, medios institucionales para ejercer el poder.

La ambición de Enrique Peña Nieto no fue ejercer una presidencia autoritaria: sus metas eran inmediatas y personales, y el narcisismo fijaba los límites de sus acciones. En más de un caso actuó por encima de sus capacidades, pero muchas de esas acciones no tuvieron verdadera trascendencia porque no tenían, y tampoco llegaron a desarrollar, un sustento institucional. Así, por ejemplo, la reforma educativa que era un capítulo central del Pacto de 2013 nunca pudo aplicarse porque no tenía el apoyo de los maestros, y el presidente no tenía los medios para obligarlos.

La huella personal que cada presidente deja en la institución y en la memoria de los ciudadanos está determinada por el juego entre sus rasgos personales y el entorno, y el uso que hace de los recursos de que dispone, su habilidad para persuadir o para negociar y, en última instancia, de su comprensión y respuesta al conjunto de reglas que norman la acción de la presidencia. Si éstas son disfuncionales o han caducado, el presidente tiene que reconocer los nuevos límites, identificar los patrones de comportamiento de los demás actores políticos, y diseñar nuevas reglas, ejercer una autolimitación que es condición de supervivencia.

La experiencia del presidente Peña Nieto es un factor de explicación de las políticas de Andrés Manuel López Obrador, que en su primer año en la presidencia de la República 
ha gobernado como si se esforzara, de forma deliberada, en anular a la institución y ejercer un poder exclusivamente personal; como si buscara construir un hiperpresidencialismo libre de restricciones institucionales.

\section{REFERENCIAS BIBLIOGRÁFICAS}

Arreola, Álvaro, "Isidro Fabela y Alfredo del Mazo Vélez (Estado de México, PRM/PRI, 1942-1951)” (pp. 31-59), en Andrew Paxman (coord.), Los gobernadores, caciques del pasado y del presente, México, Grijalbo, 2018.

Bertaccini, Tiziana y Alberto J. Aguilar Iñarritu, La transformación del PRI. De la oposición a la reconquista del poder, México, Fundación Colosio, 2015.

CAsar, María Amparo, "Quince años de gobierno sin mayoría en el Congreso mexicano”, Política y Gobierno, vol. xxv, núm. 2, 2013.

Chabat, Jorge, "La respuesta del gobierno de Calderón al desafío del narcotráfico: entre lo malo y lo peor" ( pp. 21-40), en Arturo Alvarado y Mónica Serrano (coords.), "Seguridad nacional y seguridad interior", Los grandes problemas de México, México, El Colegio de México, 2010, tomo xv.

Cheibub, José Luis; Zachary Elkins y Tom Ginsburg, "Beyond presidentialism and parliamentarism", British Journal of Political Science, vol. 44, núm. 3 (julio de 2014), pp. 515-584.

Cosío Villegas, Daniel, El estilo personal de gobernar, México, Cuadernos de Joaquín Mortiz, 1974, p. 8.

Hernández Campos, Jorge, "El fin del mito presidencial" (pp. 4755), en Enrique Florescano (coord.), Mitos mexicanos, México, Taurus, 2001, p.51.

Hernández Rodríguez, Rogelio, "La reforma interna del PRI", Foro Internacional, vol. xxxiı, núm. 2 (1991), pp. 223-249.

Hernández Rodríguez, Rogelio, "Ernesto Zedillo, la presidencia contenida”, en Foro Internacional, vol. XLıII, núm. 1 (2003), pp. 39-70. 
Hernández Rodríguez, Rogelio y Will C. Panters, "La democracia en México y el retorno del PRI”, Foro Internacional, núm. 52 (2012), pp. 755-795.

Huard, Raymond, L'éléction présidentielle au suffrage universel, París, La Dispute, 2003.

Krauze, Enrique, La presidencia imperial. Ascenso y caída del sistema político mexicano, (1940-1996), México, Tusquets, 1997.

Linz, Juan J. y Arturo Valenzuela (eds.), The Failure of Presidential Democracy, Baltimore y Londres, The Johns Hopkins University Press, 1994, 2 vols.

LoAEzA, Soledad, "La metamorfosis del Estado. Del jacobinismo centralizador a la fragmentación democrática" (pp. 23-70), en Soledad Loaeza y Jean-François Prud-homme (coords.), Los grandes problemas de México, México, El Colegio de México, 2010, tomo XIV.

LoAeza, Soledad, A la sombra de la superpotencia. Tres presidentes mexicanos en la Guerra Fría, (en prensa).

Lomnitz, Claudio, “¿Qué sucede en Veracruz?”, La Jornada, 17 de junio de 2015.

López Leyva, Miguel Armando, "El episodio reformista en México (2012-2014). Explorando las razones del cambio en la alternancia”. Estudios Políticos, $9^{a}$ época, núm. 35 (mayo-agosto de 2015), pp. 61-85.

MADrID, Miguel de la (con la colaboración de Alejandra Lajous), Cambio de rumbo, 1982-1988, México, Fondo de Cultura Económica, 2004.

Merino, Mauricio, "El precio de la Casa Blanca" (pp. 155-157), en Morena, Regalos obscenos. Lo que no pudo esconder el Pacto contra México, México, Cámara de Diputados LXXII Legislatura, 2015.

Mirón Lince, Rosa María, "Recomposición del PRI en la primera década del milenio", Convergencia, vol. 19, núm.55 (mayo-agosto de 2012), pp. 43-67.

Moreno Bagatella, Juan Carlos, "Equilibrios de poder en las entidades federativas. Estudio longitudinal de las legislaturas locales en México en el periodo 2000-2014”, Revista Mexicana de 
Análisis Político y Administración Pública, vol. III, núm. 2 (2014), pp.125-154.

Pacheco MÉndez, Guadalupe, "El PRI, relación interna de fuerzas y conflictos en la víspera del proceso electoral de 2006", Política y Gobierno, vol. xvi, núm. 1 (2009), pp. 157-190.

Panstres, Will y Rogelio Hernández Rodríguez, "La democracia mexicana y el retorno del PRI", Foro Internacional, octubre-diciembre de 2012.

Pastrana, Daniela, "Javier Duarte (Veracruz, PRI, 2010-2016). Un corazón muy chiquito”, en Revista Mexicana de Análisis Político y Administración Pública, vol. III, núm. 2 (2014), pp. 271-306.

Paxman, Andrew (coord.), Los gobernadores, caciques del pasado y del presente, México, Grijalbo, 2018.

Paxman, Andrew, "Televisa de 1997 a 2018: el socio fallido de la democratización”, en José Galindo (coord.), México contemporáneo. Aspectos económicos, políticos y sociales, Xalapa, Universidad Veracruzana, 2018.

Pérez Bárcenas, Lidia, "Higa: una historia de corrupción e impunidad" (pp. 83-134), en Morena, Regalos obscenos. Lo que no pudo esconder el Pacto contra México, México, Cámara de Diputados LXXII Legislatura, 2015.

Peterson, Mark A., "Presidential power and the potential for leadership” (pp. 363-379), en Robert Y. Shapiro, Martha Joynt Kumar y Lawrence R. Jacobs (eds.), Presidential Power. Forging the Presidency of the XXIst Century, Nueva York, Columbia University Press, 2003.

Przeworski, Adam, Crises of Democracy, Nueva York, Cambridge University Press, 2019.

Rоск L., Roberto, La historia detrás del desastre. Crónica de una herencia envenenada, México, Grijalbo, 2019, pp. 98-99.

Rodríguez García, Arturo, "Populismo en plástico", Proceso, núm. 1810, 26 de junio 2011.

SARTORI, Giovanni, Ingeniería constitucional comparada. Una investigación de estructuras, incentivos y resultados, México, Fondo de Cultura Económica, 1994.

SERrA, Gilles, "Demise and resurrection of a dominant party: Understanding the PRI's Comeback in Mexico", Journal of Politics in Latin America 5, núm. 3, diciembre de 2013, pp. 133-154. 
Skowronek, Stephen, The politics presidents make. Leadership from John Adams to Bill Clinton, Cambridge, The Belknap Press of Harvard University Press, 1993.

Tello Díaz, Carlos, "La senda del rockstar", Nexos, junio de 2012. Villamil, Jenaro, "Meganegocios, relumbrón, quimera, pobreza", Proceso, 3 de julio de 2011, proceso.com.mx 
